\title{
Extracting Atrial Activations from Intracardiac Signals during Atrial Fibrillation using Adaptive Mathematical Morphology
}

\author{
Sasan Yazdani ${ }^{1}$, Andrea Buttu ${ }^{1,2}$, Etienne Pruvot $^{2}$, Jean-Marc Vesin ${ }^{1}$, Patrizio Pascale ${ }^{2}$ \\ ${ }^{1}$ Applied Signal Processing Group, Swiss Federal Institute of Technology, Lausanne, Switzerland \\ ${ }^{2}$ Department of Cardiology, Lausanne University Hospital, Lausanne, Switzerland
}

\begin{abstract}
The detection of intracardiac activities is a major issue in the processing of atrial fibrillation signals. we evaluate a method based on mathematical morphology with an adaptive structuring element in order to extract the atrial activations from intracardiac electrograms. The structuring element is continuously updated for each activation based on the morphological characteristics of the previously detected activations. A dataset of recordings from patients with chronic atrial fibrillation who underwent catheter ablation were used in order to evaluate the performance of the proposed method. Results show high performance compared to a dataset manually annotated by an expert. The detection rate, sensitivity and positive prediction value (PPV) were respectively $99.1 \%, 99.5 \%$, 99.5\%. The proposed method is fast and offers low computational cost, which makes it a suitable approach for realtime/online scenarios.
\end{abstract}

\section{Introduction}

Intracardiac activation-time detection algorithms can be used in atrial fibrillation (AF) electrogram analysis as a first step in estimating AF characteristics. Characteristics such as cycle lengths (AFCLs) of atrial activations (AAs) can help predict persistent AF ablation outcomes [1,2]. Moreover, AFCLs can be used to determine sites with high frequency activities which may help identify critical ablation targets to restore sinus rhythm [3].

However, the varying amplitude and morphologies of AA during AF makes AA extraction difficult. Through a time consuming task, one can always measure activation intervals manually by using calipers and then averaging several measurements to find the mean AFCL. Alternatively, automatic detection methods can be used to extract AAs even though most are limited due to the use of various thresholds [4]. Spectral analysis can also help to determine AFCL by extracting AF dominant frequency (DF), i.e the frequency that has the maximum power [5]. DF methods try to approximate AA rates by a single sinusoid. Therefore, by nature, these methods are not suitable when AF electrograms present irregularities. A time domain iterative method has been proposed [6] to extract AAs from intracardiac electrograms (ICEGs). The detection threshold level is iteratively adjusted until the mean and the median of AFCL converge on a signal segment.

The purpose of this study is to evaluate a new adaptive mathematical morphology (MM) approach that extracts AAs based on their morphological features. The structuring element used in this method is adapted after each AA extraction in order to have a more reliable extraction.

\section{Methods}

\subsection{Patients and Data Acquisition}

Study Population. The study group for this research consists of three consecutive patients ( $63 \pm 1$ years) with chronic AF (sustained AF duration $17 \pm 8$ months) who underwent catheter ablation.

Electrophysiological Study. The following catheters were introduced via the femoral veins: 1) a $3.5 \mathrm{~mm}$ cooled-tip catheter for mapping and ablation (Navistar ${ }^{\circledR}$ Thermocool ${ }^{\circledR}$, Biosense Webster $\left.{ }^{\circledR}\right)$; and 2) a circumferential duodecapolar Lasso ${ }^{\circledR}$ catheter within the LA (2-6-2 25-15 mm, Biosense Webster $\left.{ }^{\circledR}\right)$. Intracardiac electrograms were continuously monitored and sampled at $2 \mathrm{kHz}(\mathrm{Ax}-$ iom Sensis $\mathrm{XP}^{\circledR}$, Siemens $\left.{ }^{\circledR}\right)$ for off-line analysis. Intracardiac EGMs were filtered with a pass band of [0.05,400] Hz. Then a notch filter at $50 \mathrm{~Hz}$ was applied to the signals in order to remove the acquisition noise. Electro-anatomical mapping and 3D reconstruction of the LA were performed with the CARTO $^{\circledR} 3$ system (Biosense Webster ${ }^{\circledR}$ ).

\subsection{Outline of the Method}

Mathematical morphology (MM). MM is a methodology proposed to extract useful topological information based on the analysis of geometrical structures. It was first 
introduced for binary images with strong set-theoric concepts and nonlinear operators, designed to extract useful information in images regarding shape and size [7]. MM is based on two elementary operators named dilation and erosion. Combining dilation and erosion leads to additional operators such as opening, closing, top-hat and bottom-hat as listed in equations 1-6. These operators are quick, simply defined and use an structuring element (SE) to extract useful information and suppress artifacts.

$$
\begin{array}{r}
\text { Dilation } \oplus: f \oplus g(n)=\max _{(1 \leq i \leq n)}\{f(i)+g(x-i)\} \\
\text { Erosion } \ominus: f \ominus g(n)=\min _{(1 \leq i \leq n)}\{f(i)-g(x-i)\} \\
\text { Open } \circ: f \circ g(n)=(f \oplus g) \ominus g(n) \\
\text { Close } \bullet: f \bullet g(n)=(f \ominus g) \oplus g(n) \\
\text { Top-Hat }: \text { THat }(g(n))=f(n)-f \circ g(n) \\
\text { Bottom-Hat }: \text { BHat }(f(n))=f(n)-f \bullet g(n)
\end{array}
$$

In these equations, $g(n)$ represents the SE with a length of $n, i$ indicates the $i$ th element of the SE, and $f$ the signal to which the MM operator is applied. Depending on the effect sought when using these operators, a specific SE must be used. Shape and length of the structuring element should be carefully chosen as they play an important role in the outcome of these operators [7,8]. For example, the average of an opening and closing of a signal with a flat SE can be used for noise suppression while the same average with a peaky SE tends to enhance peaks and valleys in the signal.

Adaptive Intracardiac MM (AIMM). AIMM is a fast MM method with an adaptive SE. It can be divided into a three-step algorithm as illustrated by the block diagram in figure 1 .

In AIMM, a non-overlapping sliding window with a length of $200 \mathrm{~ms}$ is rolled over the intracardiac signal (ICEG) at hand. In each window, the MM filter is applied on the ICEG using the current SE. Afterwards, the filtered signal called the feature signal is scrutinized in order to extract AAs. Subsequently, AAs are probed and topological features such as height and length are extracted from them in order to update the SE that is used in the MM filtering phase.

AAs have different morphologies throughout AF. Moreover, these morphologies vary with respect to the location of the catheters inside atria. Therefore, AIMM is initiated using an synthesized SE. The morphology of this synthesized SE is empirically chosen, as shown in figure 2.

The magnitude of the synthesized SE is considered as $70 \%$ of the difference between the maximum and minimum values of the first $500 \mathrm{~ms}$. Furthermore, the duration

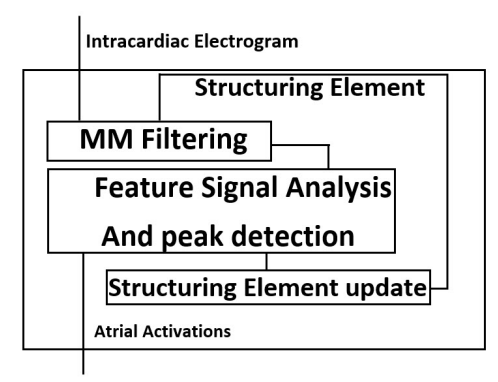

Figure 1. Block diagram of the proposed method.

of the synthesized SE is empirically chosen as $20 \mathrm{~ms}$, representing the average AA duration. The synthesized SE in AIMM comprises five points representing onset, offset, peak of AA, the minimum between the onset the peak, and the minimum between the peak and the offset.

AIMM is initiated by applying the MM filter, which uses the synthesized SE, to the first window of the ICEG. More specifically, in the MM filtering step, using Eq. 7, the topand bottom-hat operators are applied to the window and then averaged.

$$
A V G \_H a t(g(n))=f(n)-\frac{f \circ g(n)+f \bullet g(n)}{2}
$$

The peaky synthesized SE gives rise to peaks and valleys in the window and suppresses other activities. Once the MM filtering phase is carried out, the extracted feature signal is studied in order to extract AAs. In this phase, first, nonzero segments of the feature signal are extracted. Then, segments were further processed as follows:

1. Onset and offset are considered respectively as the start and end of the non-zero segment.

2. The most significant peak is extracted from the active period as AA.

3. The minimum between the onset and AA together with the minimum between AA and the offset point are extracted for the SE update phase.

Perturbations might cause non-zero feature signal values at locations where AAs do not occur. Therefore, the following physiological thresholds where used to prevent false AA extraction:

$$
A A_{\text {Valid }}=\left\{\begin{array}{l}
A A_{\text {duration }} \geq 8 \mathrm{~ms} \\
A A_{\text {candidate }}-A A_{\text {previous }} \geq 60 \mathrm{~ms}
\end{array}\right.
$$

Where $A A_{\text {candidate }}$ represents the time index of the peak of the AA candidate in the feature signal, $A A_{\text {previous }}$ the time index of the peak of the previous AA and $A A_{\text {duration }}$, the duration of the AA candidate. The last condition corresponds to the fact that cycle length of AAs are unlikely to be smaller than 60 milliseconds.

Once an AA is detected, the extracted topological features, i.e. time indices and amplitude values of the onset, 


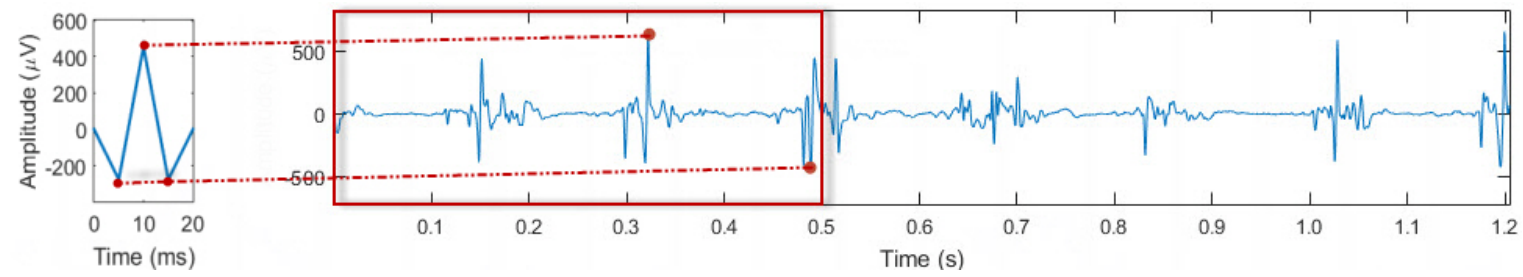

Figure 2. Synthesized structuring element and the selection of its magnitude based on the intracardiac electrogram at hand.

offset, AA peak and the two local minima are used to update the SE for further AA extraction. The SE update is applied using the following equations:

NewLoc $=(1-\alpha) \times$ Curr_Loc $+\alpha \times$ ExtractedLoc
NewAmp $=(1-\alpha) \times$ Curr_Amp $+\alpha \times$ ExtractedAmp

In these equations, $\alpha$ represents the learning coefficient and for each fiducial point, Curr_Loc and ExtractedLoc are calculated as the distance with respect to the AA onset, respectively extracted from the current $\mathrm{SE}$ and the feature signal. Furthermore, Curr_Amp and ExtractedAmp represent for each sample its amplitude in the SE and the feature signal. Using New_Loc and NewAmp, SE is updated by means of linear interpolation. The update on SE takes place after each AA extraction. Therefore, if multiple AAs are extracted from a window, SE is updated multiple times.

In order to avoid excessive changes on SE, for instance in case of large AA amplitude changes, AIMM uses variable learning coefficient to make SE adaptation flexible. Therefore, a feature called Peak Activity (PA), measured as the sum of absolute values of AA, is extracted from the feature signal. Then, after the detection of an AA, its PA is compared to that of the previous AA and the learning coefficient is updated using Eq. 11.

$$
\alpha= \begin{cases}\alpha+0.05 & \text { NewP } A<\text { Previous } P A \times 0.7 \\ \alpha-0.05 & \text { NewP } A>\text { Previous } P A \times 1.3 \\ 0.2 & \text { otherwise }\end{cases}
$$

\section{Results}

For performance evaluation, atrial activation times estimated by the adaptive MM were compared with the manual annotation provided by a blinded clinical expert. Ground truth was made of manual annotation provided by the expert. The expert was asked to annotate AAs through a graphical user interface created using MATLAB (Mathworks, Natick, MA). Furthermore, AIMM was compared against an MM method with fixed SE, and a recent stateof-the-art approach called cycle length iteration (CLI) [6]. Best performance was obtained with AIMM with a 99.1\% detection rate, $99.5 \%$ specificity and $99.5 \%$ PPV. The results are summarized in table 1. In addition, An Example of the performance of AIMM is illustrated in figure 3 and a more detailed evaluation of AIMM performance is presented in figure 4.

Table 1. Performance comparison of the proposed method on atrial activity extraction on the annotated database.

\begin{tabular}{|c|c|c|c|c|c|c|}
\hline Method & Beats & FP & FN & Detection Rate \% & Sensitivity & PPV \\
\hline AIMM & 5216 & 25 & 22 & 99.1 & 99.58 & 99.52 \\
\hline MM & 5216 & 116 & 310 & 91.83 & 94.39 & 97.82 \\
\hline CLI & 5216 & 55 & 280 & 93.58 & 94.91 & 98.96 \\
\hline
\end{tabular}

\section{Discussion}

Employing a fixed SE for AA detection may lead to unwanted activity in the feature signal which results in oversensing or under-sensing of the MM-based method. This is due to the fact that length and magnitude changes on the SE can weaken or strengthen high frequency activities which can lead to drastic effects on the feature signal $[8,9]$. Hence by adapting the SE, MM filters can become more sensitive to AAs and less to perturbations.

Our observations have shown that the best length of the $\mathrm{SE}$ is $20 \mathrm{~ms}$. Also, AIMM achieves its best performance when the ratio of the magnitude of the SE to that of the actual AA is taken as 0.7. Moreover, we examined different learning coefficients in the update phase of the AIMM in order to apply the optimal changes on the SE with respect to the activities in the ICEG. At the beginning, $\alpha$ is set to 0.5 to have a bigger impact on the synthesized SE. As more AAs are detected, the topology of the SE becomes closer to that of AAs which results in a more robust detection of the next AAs.

Complexity of AIMM consists of two elements namely, MM filtering operation complexity and the SE update phase. The total complexity can be expressed as $O(l \times$ $n)+O(l \times r)$ where $n$ represents the number of ICEG samples, $l$ the length of the structuring element and $r$ the number of detected AAs. Since $l \ll n$ and $k \ll n$ the second term can be omitted and the order of complexity of AIMM can be written as $O(n)$.

\section{Conclusion}

This paper presents a mathematical morphology approach with an adaptive structuring element to detect atrial 


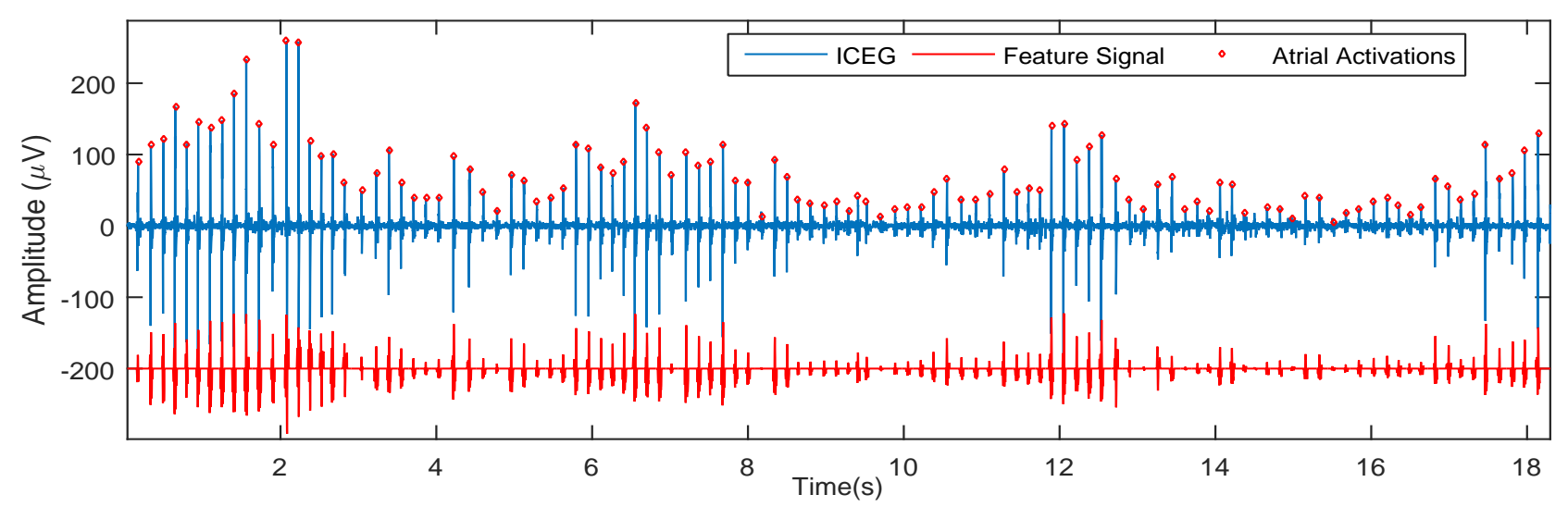

Figure 3. Performance of the proposed method on a low quality intracardiac electrogram.

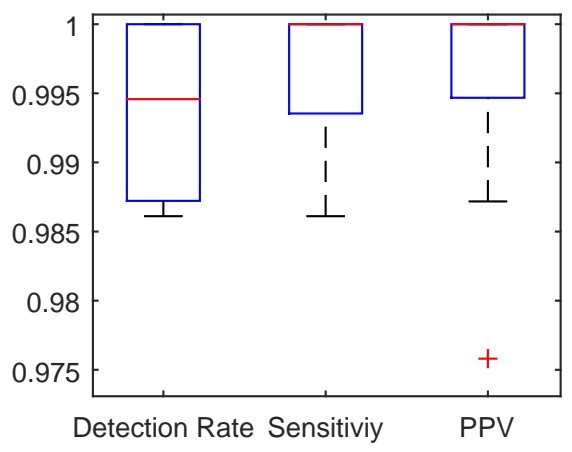

Figure 4. Performance box plot of the proposed method.

activations in intracardiac signals. The adaptive structuring element is updated after the detection of AAs in order to provide robust and accurate extraction. Results suggest that the adaptive structuring element can efficiently estimate atrial activation times. AIMM works on an activation-to-activation basis, avoids excessive use of arbitrary thresholds, and incorporates physiological constraints. Furthermore, AIMM is fast and offers low computational cost. These characteristics of AIMM makes it a viable approach for real-time/online scenarios.

\section{Acknowledgements}

This study was performed in the framework of the NanoTera initiative supported by the Swiss National Science Foundation (SNSF).

\section{References}

[1] Buttu A, Forclaz A, Pascale P, Maury P, Rollin A, Vesin J, Pruvot E. Measures of right atrial organization as a means to select candidates for sinus rhythm restoration by catheter ablation. In Computing in Cardiology Conference (CinC). 2013; 1051-1054.
[2] Pascale P, Shah AJ, Roten L, Scherr D, Komatsu Y, Ramoul K, Daly M, Denis A, Derval N, Sacher F, et al. Pulmonary veins to left atrium cycle length gradient predicts procedural and clinical outcomes of persistent atrial fibrillation ablation. Circulation Arrhythmia and Electrophysiology 2014; 473-482.

[3] Nademanee K, McKenzie J, Kosar E, Schwab M, Sunsaneewitayakul B, Vasavakul T, Khunnawat C, Ngarmukos T. A new approach for catheter ablation of atrial fibrillation: mapping of the electrophysiologic substrate. Journal of the American College of Cardiology 2004;43(11):2044-2053.

[4] Berenfeld O, Ennis S, Hwang E, Hooven B, Grzeda K, Mironov S, Yamazaki M, Kalifa J, Jalife J. Time-and frequency-domain analyses of atrial fibrillation activation rate: the optical mapping reference. Heart Rhythm 2011; 8(11):1758-1765.

[5] Lin YJ, Tai CT, Kao T, Tso HW, Higa S, Tsao HM, Chang SL, Hsieh MH, Chen SA. Frequency analysis in different types of paroxysmal atrial fibrillation. Journal of the American College of Cardiology 2006;47(7):1401-1407.

[6] Ng J, Sehgal V, Ng J, Gordon D, Goldberger J. Iterative method to detect atrial activations and measure cycle length from electrograms during atrial fibrillation. IEEE Transactions on Biomedical Engineering 2014;61(2):273-278.

[7] Maragos P, Schafer RW. Morphological systems for multidimensional signal processing. Proceedings of the IEEE 1990; (4):690-710.

[8] Zhang F, Lian Y. Qrs detection based on multiscale mathematical morphology for wearable ecg devices in body area networks. IEEE Transactions on Biomedical Circuits and System 2009;(4):220-228.

[9] Yazdani S, Vesin JM. Adaptive mathematical morphology for qrs fiducial points detection in the ecg. In Computing in Cardiology Conference (CinC). 2014; 725-728.

Address for correspondence:

Sasan Yazdani

EPFL SCI STI JMV - ELD 224 - Station 11

CH-1015 Lausanne - Switzerland.

E-mail address: sasan.yazdani@epfl.ch 